\title{
MAXIMAL ACCRETIVE EXTENSIONS OF SCHRÖDINGER OPERATORS ON VECTOR BUNDLES OVER INFINITE GRAPHS
}

\author{
OGNJEN MILATOVIC AND FRANÇOISE TRUC
}

\begin{abstract}
Given a Hermitian vector bundle over an infinite weighted graph, we define the Laplacian associated to a unitary connection on this bundle and study a perturbation of this Laplacian by an operator-valued potential. We give a sufficient condition for the resulting Schrödinger operator to serve as the generator of a strongly continuous contraction semigroup in the corresponding $\ell^{p}$-space. Additionally, in the context of $\ell^{2}$-space, we study the essential self-adjointness of the corresponding Schrödinger operator.
\end{abstract}

\section{INTRODUCTION}

In recent years, there has been quite a bit of interest in the study of the Laplacian in $\ell^{p}$-spaces on infinite graphs. More precisely, let $(X, b, m)$ be a weighted graph as described in section 2.1 below, and let us define a form $Q^{(c)}$ on (complex-valued) finitely supported functions on $X$ by

$$
\begin{aligned}
Q^{(c)}(u, v) & :=\frac{1}{2} \sum_{x, y \in X} b(x, y)(u(x)-u(y))(\overline{v(x)-v(y)}) \\
& +\sum_{x \in X} w(x) u(x) \overline{v(x)}
\end{aligned}
$$

where $w: X \rightarrow[0, \infty)$. We denote by $\ell_{m}^{p}(X)$ the space of $\ell^{p}$-summable functions with weight $m$, by $Q^{(D)}$ the closure of $Q^{(c)}$ in $\ell_{m}^{2}(X)$, and by $L$ the associated self-adjoint operator. Since $Q^{(D)}$ is a Dirichlet form, the semigroup $e^{-t L}, t \geq 0$, extends to a $C_{0}$-semigroup on $\ell_{m}^{p}(X)$, where $p \in[1, \infty)$. We denote by $-L_{p}$ the generators of these semigroups. For the definition of a $C_{0}$-semigroup and its generator, see the Appendix. The following characterization of operators $L_{p}$ is given in [19]:

Assume that

$$
\sum_{n \in \mathbb{Z}_{+}} m\left(x_{n}\right)=\infty
$$

for any sequence $\left\{x_{n}\right\}_{n \in \mathbb{Z}_{+}}$of vertices such that $x_{n} \sim x_{n+1}$ for all $n \in \mathbb{Z}_{+}$. Then for any $p \in[1, \infty)$, the operator $L_{p}$ is the restriction of $\widetilde{L}$ to

$$
\operatorname{Dom}\left(L_{p}\right)=\left\{u \in \ell_{m}^{p}(X) \cap \widetilde{D_{s}}: \widetilde{L} u \in \ell_{m}^{p}(X)\right\}
$$

2010 Mathematics Subject Classification. 39A12, 35J10, 47B25.

Key words and phrases. contraction semigroup, essentially self-adjoint, infinite graph, maximal accretive, Schrödinger operator, vector bundle. 
where

$$
\widetilde{D_{s}}:=\left\{u: X \rightarrow \mathbb{C}: \sum_{y \in X} b(x, y)|u(y)|<\infty, \forall x \in X\right\},
$$

$\widetilde{L}:=\Delta_{b, m}+w / m$, and

$$
\left(\Delta_{b, m} u\right)(x):=\frac{1}{m(x)} \sum_{y \in X} b(x, y)(u(x)-u(y)) .
$$

Actually, (A1) can be replaced when $w=0$ by the existence of a compatible intrinsic metric (see [13]), or if moreover $p=2$, by the existence of an intrinsic metric so that $\frac{1}{m(x)} \sum_{y \in X} b(x, y)$ is bounded on the combinatorial neighborhood of each distance ball (see [15]).

In the case of Schrödinger operators on a Riemannian manifold $M$, it is natural to study maximal accretivity or self-adjointness properties of operators acting on sections of vector bundles over $M$. But the notion of vector bundle is also relevant on graphs; see for example [1], [11], [20], and [27. The aim of this paper is precisely to study such properties in the setup of a vector bundle over an infinite weighted graph. In particular, we give sufficient conditions for the equality of the operator $H_{p, \max }$ (vector-bundle analogue of $\left.L_{p}\right)$ and the closure in $\Gamma_{\ell_{m}^{p}}(X, F)$ (the corresponding $\ell^{p}$-space of sections of the bundle $F \rightarrow X$ ) of the restriction of $\widetilde{H}_{W, \Phi}$ (vector-bundle analogue of $\widetilde{L}$ ) to the set of finitely supported sections.

The paper is organized as follows. In sections 2.1, 2.2 and 2.3 we describe the setting: discrete sets, Hermitian vector bundle and connection, operators. The main results are presented in section 2.4, with some comments. Section 3 contains preliminary results, such as Green's formula, Kato's inequality, and ground state transform. Sections [4, 5] and 6] are devoted to the proofs of the theorems. For readers' convenience, in the Appendix we review some concepts from the theory of semigroups of operators: $C_{0}$-semigroup, generator of a $C_{0}$-semigroup, and (maximal) accretivity. Additionally, the Appendix contains the statement of Hille-Yosida Theorem and a discussion of the connection between self-adjointness and maximal accretivity of operators in Hilbert spaces.

\section{Setup And Main Results}

2.1. Weighted Graph. Let $X$ be a countably infinite set, equipped with a measure $m: X \rightarrow$ $(0, \infty)$. Let $b: X \times X \rightarrow[0, \infty)$ be a function such that

(i) $b(x, y)=b(y, x)$, for all $x, y \in X$;

(ii) $b(x, x)=0$, for all $x \in X$;

(iii) $\sum_{y \in X} b(x, y)<\infty$, for all $x \in X$.

Vertices $x, y \in X$ with $b(x, y)>0$ are called neighbors, and we denote this relationship by $x \sim y$. We call the triple $(X, b, m)$ a weighted graph. We assume that $(X, b, m)$ is connected, that is, for any $x, y \in X$ there exists a path $\gamma$ joining $x$ and $y$. Here, a path $\gamma$ is a sequence $x_{1}, x_{2}, \ldots, x_{n} \in X$ such that $x=x_{1}, y=x_{n}$, and $x_{j} \sim x_{j+1}$ for all $1 \leq j \leq n-1$. 
2.2. Hermitian Vector Bundles on Graphs and Connection. A family of (finite-dimensional) complex linear spaces $F=\bigsqcup_{x \in X} F_{x}$ is called a complex vector bundle over $X$ and written $F \rightarrow X$, if any two $F_{x}$ and $F_{y}$ are isomorphic as complex vector spaces. Then the $F_{x}$ 's are called the fibers of $F \rightarrow X$, and the complex linear space

$$
\Gamma(X, F):=\prod_{x \in X} F_{x}=\left\{u \mid u: X \rightarrow F, u(x) \in F_{x}\right\}
$$

is called the space of sections in $F \rightarrow X$. We define the space of finitely supported sections $\Gamma_{c}(X, F)$ of $F \rightarrow X$ as the set of $u \in \Gamma(X, F)$ such that $u(x)=0$ for all but finitely many $x \in X$.

Definition 2.1. An assignment $\Phi$ which associates to any $x \sim y$ an isomorphism of complex vector spaces $\Phi_{x, y}: F_{x} \rightarrow F_{y}$ is called a connection on the complex vector bundle $F \rightarrow X$ if

$$
\Phi_{y, x}=\left(\Phi_{x, y}\right)^{-1} \quad \text { for all } x \sim y .
$$

Definition 2.2. (i) A family of complex scalar products

$$
\langle\cdot, \cdot\rangle_{F_{x}}: F_{x} \times F_{x} \rightarrow \mathbb{C}, \quad x \in X,
$$

is called a Hermitian structure on the complex vector bundle $F \rightarrow X$, and the pair given by $F \rightarrow X$ and $\langle\cdot, \cdot\rangle_{F_{x}}$ is called a Hermitian vector bundle over $X$.

(ii) A connection $\Phi$ on a complex vector bundle $F \rightarrow X$ is called unitary with respect to a Hermitian structure $\langle\cdot, \cdot\rangle_{F_{x}}$ if for all $x \sim y$ one has

$$
\Phi_{x, y}^{*}=\Phi_{x, y}^{-1}
$$

where $T^{*}$ denotes the Hermitian adjoint of an operator $T: F_{x} \rightarrow F_{y}$ with respect to $\langle\cdot, \cdot\rangle_{F_{x}}$ and $\langle\cdot, \cdot\rangle_{F_{y}} \cdot$

Definition 2.3. The Laplacian $\Delta_{b, m}^{F, \Phi}: \widetilde{D} \rightarrow \Gamma(X, F)$ on a Hermitian vector bundle $F \rightarrow X$ with a unitary connection $\Phi$ is a linear operator with the domain

$$
\widetilde{D}:=\left\{u \in \Gamma(X, F): \sum_{y \in X} b(x, y)|u(y)|_{F_{y}}<\infty, \text { for all } x \in X\right\}
$$

defined by the formula

$$
\left(\Delta_{b, m}^{F, \Phi} u\right)(x)=\frac{1}{m(x)} \sum_{y \in X} b(x, y)\left(u(x)-\Phi_{y, x} u(y)\right) .
$$

Remark 2.1. The operator $\Delta_{b, m}^{F, \Phi}$ is well-defined by the property (iii) of $b(x, y)$, definition (4)), and unitarity of $\Phi$.

Remark 2.2. In the case $F_{x}=\{x\} \times \mathbb{C}$ with the canonical Hermitian structure, the sections of the bundle $F \rightarrow X$ can be canonically identified with complex-valued functions on $X$. Under this identification, any connection $\Phi$ can be uniquely written as $\Phi_{x, y}=e^{i \theta(y, x)}$, where $\theta: X \times X \rightarrow$ $[-\pi, \pi]$ is a magnetic potential on $(X, b)$, which, due to (3) , satisfies the property $\theta(x, y)=$ 
$-\theta(y, x)$ for all $x, y \in X$. As a result, we get the magnetic Laplacian operator. In particular, if $\theta \equiv 0$ we get the Laplacian operator (2).

Remark 2.3. If the property (iii) of $b(x, y)$ is replaced by

$$
\sharp\{y \in X: b(x, y)>0\}<\infty, \text { for all } x \in X,
$$

where $\sharp S$ denotes the number of elements in the set $S$, then the graph $(X, b, m)$ is called locally finite. In this case, we have $\widetilde{D}=\Gamma(X, F)$.

2.3. Operators. From now on we will always work in the setting of a Hermitian vector bundle $F \rightarrow X$ over a connected weighted graph $(X, b, m)$, equipped with a unitary connection $\Phi$.

Definition 2.4. We define the Schrödinger-type operator $\widetilde{H}_{W, \Phi}: \widetilde{D} \rightarrow \Gamma(X, F)$ by the formula

$$
\widetilde{H}_{W, \Phi} u:=\Delta_{b, m}^{F, \Phi} u+W u
$$

where $W(x): F_{x} \rightarrow F_{x}$ is a linear operator for any $x \in X$, and $\widetilde{D}$ is as in (4).

Definition 2.5. (i) For any $1 \leq p<\infty$ we denote by $\Gamma_{\ell_{m}^{p}}(X, F)$ the space of sections $u \in$ $\Gamma(X, F)$ such that

$$
\|u\|_{p}^{p}:=\sum_{x \in X} m(x)|u(x)|_{F_{x}}^{p}<\infty
$$

where $|\cdot|_{F_{x}}$ denotes the norm in $F_{x}$ corresponding to the Hermitian product $\langle\cdot, \cdot\rangle_{F_{x}}$. The space of $p$-summable functions $X \rightarrow \mathbb{C}$ with weight $m$ will be denoted by $\ell_{m}^{p}(X)$.

(ii) $B y \Gamma_{\ell^{\infty}}(X, F)$ we denote the space of bounded sections of $F$, equipped with the norm

$$
\|u\|_{\infty}:=\sup _{x \in X}|u(x)|_{F_{x}} .
$$

The space of bounded functions on $X$ will be denoted by $\ell^{\infty}(X)$.

The space $\Gamma_{\ell_{m}^{2}}(X, F)$ is a Hilbert space with the inner product

$$
(u, v):=\sum_{x \in X} m(x)\langle u(x), v(x)\rangle_{F_{x}}
$$

Definition 2.6. Let $1 \leq p<+\infty$ and let $\widetilde{D}$ be as in (4). The maximal operator $H_{p, \max }$ is given by the formula $H_{p, \max } u=\widetilde{H}_{W, \Phi} u$ with domain

$$
\operatorname{Dom}\left(H_{p, \max }\right)=\left\{u \in \Gamma_{\ell_{m}^{p}}(X, F) \cap \widetilde{D}: \widetilde{H}_{W, \Phi} u \in \Gamma_{\ell_{m}^{p}}(X, F)\right\} .
$$

Moreover if

$$
\widetilde{H}_{W, \Phi}\left[\Gamma_{c}(X, F)\right] \subseteq \Gamma_{\ell_{m}^{p}}(X, F)
$$

then we set $H_{p, \min }:=\left.\widetilde{H}_{W, \Phi}\right|_{\Gamma_{c}(X, F)}$.

Remark 2.4. Note that under our assumptions on $(X, b, m)$, the inclusion (88) does not necessarily hold. It holds if we additionally assume that $(X, b, m)$ is locally finite. 
2.4. Statement of the Results. Let us denote by $\bar{T}$ the closure of an operator $T$.

Theorem 2.1. Let $W(x): F_{x} \rightarrow F_{x}$ be a linear operator satisfying

$$
\operatorname{Re}\langle W(x) u(x), u(x)\rangle_{F_{x}} \geq 0, \quad \text { for all } x \in X .
$$

Then, the following properties hold:

(i) Let $1<p<\infty$, and assume that (8) and (A1) are satisfied. Then the operator $-\overline{H_{p, \min }}$ generates a strongly continuous contraction semigroup on $\Gamma_{\ell_{m}^{p}}(X, F)$.

(ii) Assume that (8) is satisfied for $p=1$, and that $(X, b, m)$ is stochastically complete. Then the operator $-\overline{H_{1, \min }}$ generates a strongly continuous contraction semigroup on $\Gamma_{\ell_{m}^{1}}(X, F)$.

Remark 2.5. By Definition 1.1 in [19], stochastic completeness of $(X, b, m)$ means that there is no non-trivial and non-negative $w \in \ell^{\infty}(X)$ such that

$$
\left(\Delta_{b, m}+\alpha\right) w \leq 0, \quad \alpha>0,
$$

where $\Delta_{b, m}$ is as in (2).

Remark 2.6. The notions of generator of a strongly continuous semigroup and (maximal) accretivity are reviewed in the Appendix. In particular, under the assumptions of Theorem 2.1, the operator $\overline{H_{p, \min }}$ is maximal accretive for all $1 \leq p<\infty$.

In the next theorem, we make the following assumption, which is stronger than (8):

$$
\widetilde{H}_{W, \Phi}\left[\Gamma_{c}(X, F)\right] \subseteq \Gamma_{\ell_{m}^{p}}(X, F) \cap \Gamma_{\ell_{m}^{p^{*}}}(X, F),
$$

with $1 / p+1 / p^{*}=1$.

Remark 2.7. If $(X, b, m)$ is a locally finite graph then (10) is satisfied. If $\inf _{x \in X} m(x)>0$ then (A1) and (10) are satisfied.

Theorem 2.2. Assume that the hypotheses (A1) and (9) are satisfied. Then, the following properties hold:

(i) Let $1<p<\infty$, and assume that (10) is satisfied. Then $\overline{H_{p, \min }}=H_{p, \max }$.

(ii) Assume that (10) is satisfied for $p=1$, and that $(X, b, m)$ is stochastically complete. Then $\overline{H_{1, \min }}=H_{1, \max }$.

Regarding self-adjointness problems, let us point out that the results of [3, 4, 21, 24, 25] and Theorem 5 in [18] can be extended to the vector-bundle setting. As an illustration, we state and prove an extension of Theorem 1.5 from [25]. Before doing this, we recall the notion of intrinsic metric.

Definition 2.7. A pseudo metric is a map $d: X \times X \rightarrow[0, \infty)$ such that $d(x, y)=d(y, x)$, for all $x, y \in X ; d(x, x)=0$, for all $x \in X$; and $d(x, y)$ satisfies the triangle inequality. A pseudo metric $d=d_{\sigma}$ is called a path pseudo metric if there exists a map $\sigma: X \times X \rightarrow[0, \infty)$ such that $\sigma(x, y)=\sigma(y, x)$, for all $x, y \in X ; \sigma(x, y)>0$ if and only if $x \sim y$; and $d_{\sigma}(x, y)=$ 
$\inf \left\{l_{\sigma}(\gamma): \gamma\right.$ path connecting $x$ and $\left.y\right\}$, where the length $l_{\sigma}$ of the path $\gamma=\left(x_{0}, x_{1}, \ldots, x_{n}\right)$ is given by

$$
l_{\sigma}(\gamma)=\sum_{i=0}^{n-1} \sigma\left(x_{i}, x_{i+1}\right)
$$

On a locally finite graph a path pseudo metric is a metric; see [15].

Definition 2.8. A pseudo metric d on $(X, b, m)$ is called intrinsic if

$$
\frac{1}{m(x)} \sum_{y \in X} b(x, y)(d(x, y))^{2} \leq 1, \quad \text { for all } x \in X .
$$

Remark 2.8. The concept of intrinsic pseudo metric goes back to [9] which discusses a more general situation. For graphs it has been discussed in [14] and [8]. Related earlier material can be found in $[22]$.

We will also use the notion of a regular graph introduced in [3], which is a (not yet published) revised version of [2]. Let us first recall the definition of the boundary of a given set $A \subseteq X$ :

$$
\partial A:=\{x \in A \text { : there exists } y \in X \backslash A \text { such that } y \sim x\} .
$$

In the sequel, we denote by $(\widehat{X}, \widehat{d})$ the metric completion of $(X, d)$, and we define the Cauchy boundary $X_{\infty}$ as follows: $X_{\infty}:=\widehat{X} \backslash X$. Note that $(X, d)$ is metrically complete if and only if $X_{\infty}$ is empty. For a path metric $d=d_{\sigma}$ on $X$ and $x \in X$, we set

$$
D(x):=\inf _{z \in X_{\infty}} \widehat{d}_{\sigma}(x, z) \text {. }
$$

Definition 2.9. Let $(X, b, m)$ be a graph with a path metric $d_{\sigma}$. Let $\varepsilon>0$ be given and let

$$
X_{\varepsilon}:=\{x \in X: D(x) \geq \varepsilon\} .
$$

We say that $(X, b, m)$ is regular if for any sufficiently small $\varepsilon$, any bounded subset of $\partial X_{\varepsilon}$ (for the metric $\left.d_{\sigma}\right)$ is finite.

Remark 2.9. Metrically complete graphs $(X, d)$ are regular since $D(x)=\infty$ for any $x \in X$, which implies that $X_{\varepsilon}=X$, so that $\partial X_{\varepsilon}=\emptyset$.

Remark 2.10. Definition 2.9 covers also a broad class of metrically non-complete graphs. For instance, weighted graphs whose first Betti number is finite are regular. In particular, any weighted tree is regular; see [3].

Theorem 2.3. Let $(X, b, m)$ be a locally finite graph with an intrinsic path metric $d=d_{\sigma}$. Assume that $(X, b, m)$ is regular. Let $W(x): F_{x} \rightarrow F_{x}$ be a linear self-adjoint operator such that there exists a constant $C$ satisfying

$$
\langle W(x) u(x), u(x)\rangle_{F_{x}} \geq\left(\frac{1}{2(D(x))^{2}}-C\right)|u(x)|_{F_{x}}^{2},
$$

for all $x \in X$ and all $u \in \Gamma_{c}(X, F)$, where $D(x)$ is as in (11). Then $\widetilde{H}_{W, \Phi}$ is essentially self-adjoint on $\Gamma_{c}(X, F)$. 


\section{Preliminary Lemmas}

3.1. Green's Formula. We now give a variant of Green's formula, which is analogous to Lemma 2.1 in [10] and Lemma 4.7 in [12].

Notation 3.1. Let $W(x): F_{x} \rightarrow F_{x}$ be a linear operator. We denote by $W^{*}$ the Hermitian adjoint of $W$, that is, $(W(x))^{*}$ is the Hermitian adjoint of $W(x)$ with respect to $\langle\cdot, \cdot\rangle_{F_{x}}$.

Lemma 3.1. Let $\widetilde{H}_{W, \Phi}$ be as in (6). The following properties hold:

(i) if $\widetilde{H}_{W, \Phi}\left[\Gamma_{c}(X, F)\right] \subseteq \Gamma_{\ell_{m}^{p}}(X, F)$ for some $1 \leq p \leq \infty$, then any $u \in \Gamma_{\ell_{m}^{p^{*}}}(X, F)$ with $1 / p+1 / p^{*}=1$ belongs to the set $\widetilde{D}$ defined by (4);

(ii) for all $u \in \widetilde{D}$ and all $v \in \Gamma_{c}(X, F)$, the sums

$$
\sum_{x \in X} m(x)\left\langle\widetilde{H}_{W, \Phi} u, v\right\rangle_{F_{x}}, \quad \sum_{x \in X} m(x)\left\langle u, \widetilde{H}_{W^{*}, \Phi} v\right\rangle_{F_{x}},
$$

and the expression

$$
\begin{aligned}
& \frac{1}{2} \sum_{x, y \in X} b(x, y)\left\langle u(x)-\Phi_{y, x} u(y), v(x)-\Phi_{y, x} v(y)\right\rangle_{F_{x}} \\
& +\sum_{x \in X} m(x)\langle W(x) u(x), v(x)\rangle_{F_{x}}
\end{aligned}
$$

converge absolutely and agree.

Proof. To make the notations simpler, throughout the proof we suppress $F_{x}$ in $|\cdot|_{F_{x}}$. From the assumption $\widetilde{H}_{W, \Phi}\left[\Gamma_{c}(X, F)\right] \subseteq \Gamma_{\ell_{m}^{p}}(X, F)$, it is easily seen that the function $y \mapsto b(x, y) / m(y)$ belongs to $\ell_{m}^{p}(X)$, for all $x \in X$. In the case $1<p^{*}<\infty$, for all $u \in \Gamma_{\ell_{m}^{p^{*}}}(X, F)$, by Hölder's inequality with $1 / p+1 / p^{*}=1$ we have

$$
\sum_{y \in X} b(x, y)|u(y)| \leq\left(\sum_{y \in X}\left(\frac{b(x, y)}{m(y)}\right)^{p} m(y)\right)^{1 / p}\left(\sum_{y \in X}|u(y)|^{p^{*}} m(y)\right)^{1 / p^{*}} .
$$

In the case $p^{*}=1$, for all $u \in \Gamma_{\ell_{m}^{1}}(X, F)$, by Hölder's inequality with $p=\infty$ and $p^{*}=1$ we have

$$
\sum_{y \in X} b(x, y)|u(y)| \leq \sup _{y \in X}\left(\frac{b(x, y)}{m(y)}\right)\left(\sum_{y \in X}|u(y)| m(y)\right) .
$$

In the case $p^{*}=\infty$, for all $u \in \Gamma_{\ell}(X, F)$, by Hölder's inequality with $p=1$ and $p^{*}=\infty$ we have

$$
\sum_{y \in X} b(x, y)|u(y)| \leq \sup _{y \in X}(|u(y)|)\left(\sum_{y \in X} b(x, y)\right) .
$$

This concludes the proof of property (i). Let us prove property (ii). Since $v \in \Gamma_{c}(X, F)$, the first sum is performed over finitely many $x \in X$. Hence, this sum converges absolutely. The 
proof of absolute convergence of the second sum and the expression (14) is based on the next two estimates. By Cauchy-Schwarz inequality and unitarity of $\Phi_{y, x}$ we get

$$
\sum_{x, y \in X}\left|b(x, y)\left\langle u(x), \Phi_{y, x} v(y)\right\rangle_{F_{x}}\right| \leq \sum_{y \in X}|v(y)|\left(\sum_{x \in X} b(x, y)|u(x)|\right)<\infty,
$$

where the convergence follows from the fact that $u \in \widetilde{D}$ and $v \in \Gamma_{c}(X, F)$. Similarly,

$$
\sum_{x, y \in X}\left|b(x, y)\langle u(x), v(x)\rangle_{F_{x}}\right| \leq \sum_{x \in X}|u(x)||v(x)|\left(\sum_{y \in X} b(x, y)\right)<\infty
$$

where the convergence follows by property (iii) of $b(x, y)$ and since $v \in \Gamma_{c}(X, F)$. The equality of the three sums follows directly from Fubini's theorem. This shows property (ii).

3.2. Kato's Inequality. This version of Kato's inequality extends that of [6].

Lemma 3.2. Let $\Delta_{b, m}$ and $\Delta_{b, m}^{F, \Phi}$ be defined as in (2) and (5) respectively. Then, the following pointwise inequality holds for all $u \in \widetilde{D}$ :

$$
|u|\left(\Delta_{b, m}|u|\right) \leq \operatorname{Re}\left\langle\Delta_{b, m}^{F, \Phi} u, u\right\rangle_{F_{x}},
$$

where $|\cdot|$ denotes the norm in $F_{x}$, and Re $z$ denotes the real part of a complex number $z$.

Proof. Using (2), (5), and the unitarity of $\Phi_{y, x}$, we obtain

$|u(x)|\left(\left(\Delta_{b, m}|u|\right)(x)\right)-\operatorname{Re}\left\langle\Delta_{b, m}^{F, \Phi} u(x), u(x)\right\rangle_{F_{x}}$

$=\frac{1}{m(x)} \sum_{y \in X} b(x, y)\left[\operatorname{Re}\left\langle\Phi_{y, x} u(y), u(x)\right\rangle_{F_{x}}-|u(x)||u(y)|\right] \leq 0$.

3.3. Ground State Transform. Using the definition of $\widetilde{H}_{W, \Phi}$ and unitarity of $\Phi_{y, x}$, it is easy to prove the following vector-bundle analogue of "ground state transform" from [9], [10], and [12]. We omit the proof here.

Lemma 3.3. Assume that $W(x): F_{x} \rightarrow F_{x}$ is a self-adjoint operator. Assume that (8) is satisfied for $p=2$. Let $\lambda \in \mathbb{R}$, and let $u \in \widetilde{D}$ so that

$$
\left(\widetilde{H}_{W, \Phi}-\lambda\right) u=0 \text {. }
$$

Then, for all finitely supported functions $g: X \rightarrow \mathbb{R}$, we have

$$
\left(\left(\widetilde{H}_{W, \Phi}-\lambda\right)(g u), g u\right)=\frac{1}{2} \sum_{x, y \in X} b(x, y)(g(x)-g(y))^{2}\left(R e\left\langle u(x), \Phi_{y, x} u(y)\right\rangle_{F_{x}}\right) .
$$

\section{Proof of Theorem 2.1}

In Lemmas 4.1 and 4.3 below, we assume that the hypotheses of Theorem 2.1 are satisfied.

Lemma 4.1. Let $1 \leq p<\infty$. Then, the operator $H_{p, \min }$ satisfies the following inequality for all $u \in \Gamma_{c}(X, F)$ :

$$
R e \sum_{x \in X} m(x)\left\langle\left(H_{p, \min } u\right)(x), u(x)|u(x)|^{p-2}\right\rangle_{F_{x}} \geq 0
$$


Proof. Let $u \in \Gamma_{c}(X, F)$ be arbitrary. By Lemma 3.1(ii) with $W=0, u \in \Gamma_{c}(X, F)$ and $v:=u|u|^{p-2}$, we have

$$
\begin{aligned}
& \operatorname{Re} \sum_{x \in X} m(x)\left\langle\left(\Delta_{b, m}^{F, \Phi} u\right)(x), u(x)|u(x)|^{p-2}\right\rangle_{F_{x}}=\frac{1}{2} \sum_{x, y \in X} b(x, y)\left[|u(x)|^{p}\right. \\
& \left.+|u(y)|^{p}-\operatorname{Re}\left\langle\Phi_{y, x} u(y), u(x)|u(x)|^{p-2}\right\rangle_{F_{x}}-\operatorname{Re}\left\langle\Phi_{x, y} u(x), u(y)|u(y)|^{p-2}\right\rangle_{F_{y}}\right] \\
& \geq \frac{1}{2} \sum_{x, y \in X} b(x, y)\left[|u(x)|^{p}+|u(y)|^{p}-|u(x)||u(y)|^{p-1}\right. \\
& \left.-|u(y)||u(x)|^{p-1}\right] .
\end{aligned}
$$

For $p=1$, from (17) and the assumption (9) we easily get (16).

Let $1<p<\infty$ and let $p^{*}$ satisfy $1 / p+1 / p^{*}=1$. By Young's inequality we have

$$
|u(x)||u(y)|^{p-1} \leq \frac{|u(x)|^{p}}{p}+\frac{\left(|u(y)|^{p-1}\right)^{p^{*}}}{p^{*}}=\frac{|u(x)|^{p}}{p}+\frac{(p-1)|u(y)|^{p}}{p}
$$

and, likewise,

From the last two inequalities we get

$$
|u(y)||u(x)|^{p-1} \leq \frac{|u(y)|^{p}}{p}+\frac{(p-1)|u(x)|^{p}}{p} .
$$

$$
-|u(x)||u(y)|^{p-1}-|u(y)||u(x)|^{p-1} \geq-|u(x)|^{p}-|u(y)|^{p} .
$$

Using (18), (17), and the assumption (9), we obtain (16).

The following lemma is a special case of Proposition 8 in [18]:

Lemma 4.2. Assume (A1). Let $\alpha>0$ and $1 \leq p<\infty$. Let $\Delta_{b, m}$ be as in (2). Assume that $u \in \ell_{m}^{p}(X)$ is a real-valued function satisfying the inequality $\left(\Delta_{b, m}+\alpha\right) u \geq 0$. Then $u \geq 0$.

Remark 4.1. The case $p=\infty$ is more complicated and involves the notion of stochastic completeness; see, for instance, [14, [18, [19].

In the remainder of this section and in section 5, we will use certain arguments of Section A in [17] and [23] in our setting. In the sequel, Ran $T$ denotes the range of an operator $T$.

Lemma 4.3. Let $1<p<\infty$ and let $\lambda \in \mathbb{C}$ with Re $\lambda>0$. Then, Ran $\left(H_{p, \min }+\lambda\right)$ is dense in $\ell_{m}^{p}(X)$.

Proof. Let $u \in\left(\Gamma_{\ell_{m}^{p}}(X, F)\right)^{*}=\Gamma_{\ell_{m}^{p^{*}}}(X, F)$, be a continuous linear functional that annihilates $\left(\lambda+H_{p, \min }\right) \Gamma_{c}(X, F)$ :

$$
\sum_{x \in X} m(x)\left\langle\left(\lambda+H_{p, \min }\right) v(x), u(x)\right\rangle_{F_{x}}=0, \quad \text { for all } v \in \Gamma_{c}(X, F) .
$$

By assumption (8) we know that $\widetilde{H}_{W, \Phi} v \in \Gamma_{\ell_{m}^{p}}(X, F)$. Since $u \in \Gamma_{\ell_{m}^{p^{*}}}(X, F)$, by Lemma 3.1(i) we have $u \in \widetilde{D}$. Now using Lemma 3.1(ii) in (19), we get

$$
\sum_{x \in X} m(x)\left\langle v(x),\left(\bar{\lambda}+\widetilde{H}_{W^{*}, \Phi}\right) u(x)\right\rangle_{F_{x}}=0, \quad \text { for all } v \in \Gamma_{c}(X, F),
$$


where $\bar{\lambda}$ is the complex conjugate of $\lambda$. The last equality leads to

$$
\left(\bar{\lambda}+\Delta_{b, m}^{F, \Phi}+W^{*}\right) u=0 .
$$

Using Kato's inequality (15), assumption (9), and (20) we have

$$
\begin{aligned}
& |u|\left(\Delta_{b, m}|u|\right) \leq \operatorname{Re}\left\langle\Delta_{b, m}^{F, \Phi} u, u\right\rangle_{F_{x}} \\
& =-(\operatorname{Re} \lambda)|u|^{2}-\operatorname{Re}\left\langle W^{*} u, u\right\rangle_{F_{x}} \leq-(\operatorname{Re} \lambda)|u|^{2},
\end{aligned}
$$

where $|u| \in \ell_{m}^{p^{*}}(X)$ with $1<p^{*}<\infty$. Rewriting the last inequality, we obtain

$$
|u|\left(\Delta_{b, m}|u|+(\operatorname{Re} \lambda)|u|\right) \leq 0 .
$$

For all $x \in X$ such that $u(x) \neq 0$, we may divide both sides of the last inequality by $|u(x)|$ to get

$$
\left(\Delta_{b, m}+\operatorname{Re} \lambda\right)|u| \leq 0 .
$$

Note that the inequality (21) also holds for those $x \in X$ such that $u(x)=0$; in this case, the left hand side of (21) is non-positive by (2). Thus, the inequality (21) holds for all $x \in X$. By Lemma 4.2, from (21) we get $|u| \leq 0$. Hence, $u=0$.

End of the Proof of Theorem 2.1(i). The inequality (16) means that $H_{p, \text { min }}$ is accretive in $\Gamma_{\ell_{m}^{p}}(X, F)$; see (R1) in the Appendix with $j(u)=u|u|^{p-2}$. Hence, $H_{p, \min }$ is closable and $\overline{H_{p, \min }}$ is accretive in $\Gamma_{\ell_{m}^{p}}(X, F)$; see the Appendix. Therefore, for all $u \in \operatorname{Dom}\left(\overline{H_{p, \min }}\right)$ the following inequality holds:

$$
\operatorname{Re} \sum_{x \in X} m(x)\left\langle\left(H_{p, \min } u\right)(x), u(x)|u(x)|^{p-2}\right\rangle_{F_{x}} \geq 0 .
$$

Let $\lambda \in \mathbb{C}$ with $\operatorname{Re} \lambda>0$. Using Hölder's inequality, from (22) we get

$$
(\operatorname{Re} \lambda)\|u\|_{p} \leq\left\|\left(\lambda+\overline{H_{p, \min }}\right) u\right\|_{p},
$$

for all $u \in \operatorname{Dom}\left(\overline{H_{p, \min }}\right)$. By Lemma 4.3 we know that Ran $\left(H_{p, \min }+\lambda\right)$ is dense in $\Gamma_{\ell_{m}^{p}}(X, F)$. This, together with (23) , shows that $\operatorname{Ran}\left(\overline{H_{p, \min }}+\lambda\right)=\Gamma_{\ell_{m}^{p}}(X, F)$. Hence, from (23) we get

$$
\left\|\left(\xi+\overline{H_{p, \min }}\right)^{-1}\right\| \leq \frac{1}{\xi}, \quad \text { for all } \xi>0,
$$

where $\|\cdot\|$ is the operator norm $\Gamma_{\ell_{m}^{p}}(X, F) \rightarrow \Gamma_{\ell_{m}^{p}}(X, F)$. Thus, $-\overline{H_{p, \min }}$ satisfies the conditions (C1), (C2) and (C3) of Hille-Yosida Theorem; see the Appendix. Hence, $-\overline{H_{p, \min }}$ is the generator of a strongly continuous contraction semigroup on $\Gamma_{\ell_{m}^{p}}(X, F)$.

Proof of Theorem 2.1(ii). Repeating the proof of Lemma 4.3 in the case $p=1$ and using Remark 2.5, from (21) with $u \in \Gamma_{\ell^{\infty}}(X, F)$ we obtain $|u|=0$. Therefore, for all $\lambda \in \mathbb{C}$ with $\operatorname{Re} \lambda>0$, the set $\operatorname{Ran}\left(H_{1, \min }+\lambda\right)$ is dense in $\Gamma_{\ell_{m}^{1}}(X, F)$. From here on, we may repeat the proof of Theorem 2.1(i). 


\section{Proof of Theorem 2.2}

We begin with the following lemma.

Lemma 5.1. Let $1 \leq p<\infty$ and $1 / p+1 / p^{*}=1$. Assume that (10) is satisfied. Then $H_{p, \max }$ is a closed operator.

Proof. Let $u_{k}$ be a sequence of elements in $\operatorname{Dom}\left(H_{p, \max }\right)$ such that $u_{k} \rightarrow u$ and $H_{p, \max } u_{k} \rightarrow f$, as $k \rightarrow \infty$, using the norm convergence in $\Gamma_{\ell_{m}^{p}}(X, F)$. We need to show that $u \in \operatorname{Dom}\left(H_{p, \max }\right)$ and $f=H_{p, \max } u$. Let $v \in \Gamma_{c}(X, F)$ be arbitrary, and consider the sum

$$
\sum_{x \in X} m(x)\left\langle\left(H_{p, \max } u_{k}\right)(x), v(x)\right\rangle_{F_{x}}=\sum_{x \in X} m(x)\left\langle\left(\widetilde{H}_{W, \Phi} u_{k}\right)(x), v(x)\right\rangle_{F_{x}} .
$$

By Lemma 3.1(ii) we have

$$
\sum_{x \in X} m(x)\left\langle\left(\widetilde{H}_{W, \Phi} u_{k}\right)(x), v(x)\right\rangle_{F_{x}}=\sum_{x \in X} m(x)\left\langle u_{k}(x),\left(\widetilde{H}_{W^{*}, \Phi} v\right)(x)\right\rangle_{F_{x}} .
$$

Using the norm convergence $u_{k} \rightarrow u$ in $\Gamma_{\ell_{m}^{p}}(X, F)$ and the assumption $\widetilde{H}_{W, \Phi} v \in \Gamma_{\ell_{m}^{p^{*}}}(X, F)$ with $1 / p+1 / p^{*}=1$, by Hölder's inequality we get

$$
\sum_{x \in X} m(x)\left\langle u_{k}(x),\left(\widetilde{H}_{W^{*}, \Phi} v\right)(x)\right\rangle_{F_{x}} \rightarrow \sum_{x \in X} m(x)\left\langle u(x),\left(\widetilde{H}_{W^{*}, \Phi} v\right)(x)\right\rangle_{F_{x}} .
$$

Using the norm convergence $\widetilde{H}_{W, \Phi} u_{k} \rightarrow f$ in $\Gamma_{\ell_{m}^{p}}(X, F)$, by Hölder's inequality we get

$$
\sum_{x \in X} m(x)\left\langle\left(\widetilde{H}_{W, \Phi} u_{k}\right)(x), v(x)\right\rangle_{F_{x}} \rightarrow \sum_{x \in X} m(x)\langle f(x), v(x)\rangle_{F_{x}} .
$$

Therefore, taking the limit as $k \rightarrow \infty$ on both sides of (24), we obtain

$$
\sum_{x \in X} m(x)\left\langle u(x),\left(\widetilde{H}_{W^{*}, \Phi} v\right)(x)\right\rangle_{F_{x}}=\sum_{x \in X} m(x)\langle f(x), v(x)\rangle_{F_{x}} .
$$

Since $u \in \Gamma_{\ell_{m}^{p}}(X, F)$ and since $\widetilde{H}_{W, \Phi}\left[\Gamma_{c}(X, F)\right] \subseteq \Gamma_{\ell_{m}^{p^{*}}}(X, F)$, we may use Lemma 3.1(i) to conclude $u \in \widetilde{D}$. Using Lemma 3.1(ii), we rewrite the left-hand side of (25) as follows:

$$
\sum_{x \in X} m(x)\left\langle u(x),\left(\widetilde{H}_{W^{*}, \Phi} v\right)(x)\right\rangle_{F_{x}}=\sum_{x \in X} m(x)\left\langle\left(\widetilde{H}_{W, \Phi} u\right)(x), v(x)\right\rangle_{F_{x}} .
$$

Since $v \in \Gamma_{c}(X, F)$ is arbitrary, by (25) and (26) we get $\widetilde{H}_{W, \Phi} u=f$. Thus, $u \in \operatorname{Dom}\left(H_{p, \max }\right)$ and $H_{p, \max } u=f$. Therefore, $H_{p, \max }$ is closed.

Maximal Operator Associated with $\Delta_{b, m}$. Let $1 \leq p<\infty$ and let $\Delta_{b, m}$ be as in (2). We define the maximal operator $L_{p, \max }$ in $\ell_{m}^{p}(X)$ by the formula $L_{p, \max } u=\Delta_{b, m} u$ with the domain

$$
\operatorname{Dom}\left(L_{p, \max }\right)=\left\{u \in \ell_{m}^{p}(X) \cap \widetilde{D}: \Delta_{b, m} u \in \ell_{m}^{p}(X)\right\}
$$

where $\widetilde{D}$ is as in (4) and sections are replaced by functions $X \rightarrow \mathbb{C}$. 
Under the assumption (A1), it is known that $-L_{p \text {,max }}$ generates a strongly continuous contraction semigroup on $\ell_{m}^{p}(X)$ for all $1 \leq p<\infty$; see Theorem 5 in [19]. Thus, by Hille-Yosida Theorem (see the Appendix), we have

$$
(0, \infty) \subset \rho\left(-L_{p, \max }\right) \quad \text { and } \quad\left\|\left(\xi+L_{p, \max }\right)^{-1}\right\| \leq \frac{1}{\xi},
$$

for all $\xi>0$, where $\rho(T)$ denotes the resolvent set of an operator $T$.

Lemma 5.2. Let $1 \leq p<\infty$ and let $\lambda \in \mathbb{C}$ with Re $\lambda>0$. Assume that the hypotheses (A1) and (9) are satisfied. Then, the following properties hold:

(i) for all $u \in \operatorname{Dom}\left(H_{p, \max }\right)$, we have

$$
(\operatorname{Re} \lambda)\|u\|_{p} \leq\left\|\left(\lambda+H_{p, \max }\right) u\right\|_{p}
$$

(ii) the operator $\lambda+H_{p, \max }: \operatorname{Dom}\left(H_{p, \max }\right) \subset \Gamma_{\ell_{m}^{p}}(X, F) \rightarrow \Gamma_{\ell_{m}^{p}}(X, F)$ is injective.

Proof. Let $u \in \operatorname{Dom}\left(H_{p, \text { max }}\right)$ and $f:=\left(\lambda+H_{p, \text { max }}\right) u$. By the definition of $\operatorname{Dom}\left(H_{p, \text { max }}\right)$, we have $f \in \Gamma_{\ell_{m}^{p}}(X, F)$, where $1<p<+\infty$. Using (15) and (99) we get

$$
\begin{aligned}
& |u|\left(\left(\operatorname{Re} \lambda+\Delta_{b, m}\right)|u|\right) \leq \operatorname{Re}\left\langle\left(\lambda+\Delta_{b, m}^{F, \Phi}\right) u, u\right\rangle_{F_{x}} \\
& \leq \operatorname{Re}\left\langle\left(\lambda+\Delta_{b, m}^{F, \Phi}+W\right) u, u\right\rangle_{F_{x}}=\operatorname{Re}\langle f, u\rangle_{F_{x}} \leq|f||u| .
\end{aligned}
$$

In what follows, we denote $\xi:=\operatorname{Re} \lambda$. For all $x \in X$ such that $u(x) \neq 0$, we may divide both sides of the last inequality by $|u(x)|$ to get

$$
\left(\xi+\Delta_{b, m}\right)|u| \leq|f| .
$$

Note that the inequality (29) also holds for those $x \in X$ such that $u(x)=0$; in this case, the left hand side of (29) is non-positive by (2). Thus, the inequality (29) holds for all $x \in X$.

According to (27) the linear operator

$$
\left(\xi+L_{p, \max }\right)^{-1}: \ell_{m}^{p}(X) \rightarrow \ell_{m}^{p}(X)
$$

is bounded. Hence, we can rewrite (29) as

$$
\left(\xi+\Delta_{b, m}\right)\left[\left(\xi+L_{p, \max }\right)^{-1}|f|-|u|\right] \geq 0 .
$$

Since

$$
\left(\xi+L_{p, \max }\right)^{-1}|f| \in \ell_{m}^{p}(X) \quad \text { and } \quad|u| \in \ell_{m}^{p}(X),
$$

it follows that $\left(\left(\xi+L_{p, \max }\right)^{-1}|f|-|u|\right) \in \ell_{m}^{p}(X)$. Hence, applying Lemma 4.2 to (30) we get

$$
|u| \leq\left(\xi+L_{p, \max }\right)^{-1}|f| \text {. }
$$

Taking the $\ell^{p}$-norms on both sides and using (27) we get

$$
\|u\|_{p} \leq\left\|\left(\xi+L_{p, \max }\right)^{-1}|f|\right\|_{p} \leq \frac{1}{\xi}\|f\|_{p}
$$

and (28) is proven. We turn to property (ii). Assume that $u \in \operatorname{Dom}\left(H_{p, \max }\right)$ and $\left(\lambda+H_{p, \max }\right) u=$ 0 . Using (28) we get $\|u\|_{p}=0$, and hence $u=0$. This shows that $\lambda+H_{p \text {,max }}$ is injective. 
End of the Proof of Theorem 2.2. We will consider the cases $1<p<\infty$ and $p=1$ simultaneously, keeping in mind the stochastic completeness assumption on $(X, b, m)$ when $p=1$. Since $H_{p, \text { min }} \subset H_{p, \max }$ and since $H_{p, \max }$ is closed (see Lemma 5.1), it follows that $\overline{H_{p, \min }} \subset$ $H_{p, \max }$. To prove the equality $\overline{H_{p, \min }}=H_{p, \max }$, it is enough to show that $\operatorname{Dom}\left(H_{p, \max }\right) \subset$ $\operatorname{Dom}\left(\overline{H_{p, \min }}\right)$. Let $\xi>0$, let $u \in \operatorname{Dom}\left(H_{p, \max }\right)$, and consider

$$
v:=\left(\overline{H_{p, \min }}+\xi\right)^{-1}\left(H_{p, \max }+\xi\right) u .
$$

By Theorem 2.1, the element $v$ is well-defined, and $v \in \operatorname{Dom}\left(\overline{H_{p, \min }}\right)$.

Since $\overline{H_{p, \text { min }}} \subset H_{p \text {, max }}$, from (31) we get

$$
\left(H_{p, \max }+\xi\right)(v-u)=0 .
$$

Since $H_{p, \max }+\xi$ is an injective operator (see Lemma 5.2), we get $v=u$. Therefore, $u \in$ $\operatorname{Dom}\left(\overline{H_{p, \min }}\right)$.

\section{Proof of Theorem 2.3}

The following lemma, whose proof is given in Proposition 4.1 of [3], describes an important property of regular graphs. For the case of metrically complete graphs, see [15].

Lemma 6.1. Assume that $(X, b, m)$ is a locally finite graph with a path metric $d_{\sigma}$. Additionally, assume that $(X, b, m)$ is regular in the sense of Definition [2.9. Let $X_{\varepsilon}$ be as in (12). Then, closed and bounded subsets of $X_{\varepsilon}$ are finite.

By Remark 2.4 and Lemma 3.1(ii), $\left.\widetilde{H}_{W, \Phi}\right|_{\Gamma_{c}(X, F)}$ is a symmetric operator in $\Gamma_{\ell_{m}^{2}}(X, F)$. To prove Theorem 2.3 we follow the method of Theorem 1.5 in [25], which goes back to [5] in the continuous setting. The main ingredient is the following Agmon-type estimate:

Lemma 6.2. Let $\lambda \in \mathbb{R}$ and let $v \in \Gamma_{\ell_{m}^{2}}(X, F)$ be a weak solution of $\left(\widetilde{H}_{W, \Phi}-\lambda\right) v=0$. Assume that there exists a constant $c_{1}>0$ such that, for all $u \in \Gamma_{c}(X, F)$

$$
\left(u,\left(\widetilde{H}_{W, \Phi}-\lambda\right) u\right) \geq \frac{1}{2} \sum_{x \in X} \max \left(\frac{1}{D(x)^{2}}, 1\right) m(x)|u(x)|_{F_{x}}^{2}+c_{1}\|u\|^{2},
$$

where $D(x)$ is as in (11). Then $v \equiv 0$.

Proof. Let $\rho$ be a number such that $0<\rho<1 / 2$. For any $\varepsilon>0$, we define $f_{\varepsilon}: X \rightarrow \mathbb{R}$ by $f_{\varepsilon}(x)=F_{\varepsilon}(D(x))$, where $D(x)$ is as in (111) and $F_{\varepsilon}: \mathbb{R}^{+} \rightarrow \mathbb{R}$ is given by $F_{\varepsilon}(s)=0$ for $s \leq \varepsilon$; $F_{\varepsilon}(s)=(s-\varepsilon) /(\rho-\varepsilon)$ for $\varepsilon \leq s \leq \rho ; F_{\varepsilon}(s)=s$ for $\rho \leq s \leq 1 ; F_{\varepsilon}(s)=1$ for $s \geq 1$.

Let us fix a vertex $x_{0}$. For any $\alpha>0$, we define $g_{\alpha}: X \rightarrow \mathbb{R}$ by $g_{\alpha}(x)=G_{\alpha}\left(d_{\sigma}\left(x_{0}, x\right)\right)$, where $G_{\alpha}: \mathbb{R}^{+} \rightarrow \mathbb{R}$ is given by $G_{\alpha}(s)=1$ for $s \leq 1 / \alpha ; G_{\alpha}(s)=-\alpha s+2$ for $1 / \alpha \leq s \leq 2 / \alpha ; G_{\alpha}(s)=0$ for $s \geq 2 / \alpha$. We also define

$$
E_{\varepsilon, \alpha}:=\left\{x \in X: \varepsilon \leq D(x) \text { and } d_{\sigma}\left(x_{0}, x\right) \leq 2 / \alpha\right\} .
$$

By Lemma 6.1 the set $E_{\varepsilon, \alpha}$ is finite because $E_{\varepsilon, \alpha}$ is a closed and bounded subset of $X_{\varepsilon}$, where $X_{\varepsilon}$ is as in (12). Since the support of $f_{\varepsilon} g_{\alpha}$ is contained in $E_{\varepsilon, \alpha}$, it follows that $f_{\varepsilon} g_{\alpha}$ is finitely supported. Using Lemma 4.1 in [2] it is easy to see that $f_{\varepsilon} g_{\alpha}$ is a $\beta$-Lipschitz function with 
respect to $d_{\sigma}$, where $\beta=\rho /(\rho-\varepsilon)+\alpha$. By Lemma 3.3 with with $g$ replaced by $f_{\varepsilon} g_{\alpha}$, unitarity of $\Phi_{y, x}, \beta$-Lipschitz property of $f_{\varepsilon} g_{\alpha}$, and Defintion 2.8, we have

$$
\left(f_{\varepsilon} g_{\alpha} v,\left(\widetilde{H}_{W, \Phi}-\lambda\right)\left(f_{\varepsilon} g_{\alpha} v\right)\right) \leq \frac{1}{2}\left(\frac{\rho}{\rho-\varepsilon}+\alpha\right)^{2} \sum_{x \in X} m(x)|v(x)|_{F_{x}}^{2} .
$$

On the other hand, by the definitions of $f_{\varepsilon}$ and $g_{\alpha}$ and the assumption (32) we have

$$
\left(f_{\varepsilon} g_{\alpha} v,\left(\widetilde{H}_{W, \Phi}-\lambda\right)\left(f_{\varepsilon} g_{\alpha} v\right)\right) \geq \frac{1}{2} \sum_{x \in S_{\rho, \alpha}} m(x)|v(x)|_{F_{x}}^{2}+c_{1}\left\|f_{\varepsilon} g_{\alpha} v\right\|^{2},
$$

where

$$
S_{\rho, \alpha}:=\left\{x \in X: \rho \leq D(x) \text { and } d_{\sigma}\left(x_{0}, x\right) \leq 1 / \alpha\right\} .
$$

Combining (34) and (33) we obtain

$$
\frac{1}{2} \sum_{x \in S_{\rho, \alpha}} m(x)|v(x)|_{F_{x}}^{2}+c_{1}\left\|f_{\varepsilon} g_{\alpha} v\right\|^{2} \leq \frac{1}{2}\left(\frac{\rho}{\rho-\varepsilon}+\alpha\right)^{2} \sum_{x \in X} m(x)|v(x)|_{F_{x}}^{2} .
$$

We fix $\rho$ and $\varepsilon$, and let $\alpha \rightarrow 0+$. After that, we let $\varepsilon \rightarrow 0+$. Finally, we take the limit as $\rho \rightarrow 0+$. As a result, we get $v \equiv 0$.

End of the Proof of Theorem 2.3. Since $\left.\Delta_{b, m}^{F, \Phi}\right|_{\Gamma_{c}(X, F)}$ is a non-negative operator, for all $u \in \Gamma_{c}(X, F)$, we have

$$
\left(u, \widetilde{H}_{W, \Phi} u\right) \geq \sum_{x \in X} m(x)\langle W(x) u(x), u(x)\rangle_{F_{x}}
$$

Therefore, using assumption (13) we obtain:

$$
\begin{aligned}
& \left(u,\left(\widetilde{H}_{W, \Phi}-\lambda\right) u\right) \geq \frac{1}{2} \sum_{x \in X} \frac{1}{D(x)^{2}} m(x)|u(x)|_{F_{x}}^{2}-(\lambda+C)\|u\|^{2} \\
& \geq \frac{1}{2} \sum_{x \in X} \max \left(\frac{1}{D(x)^{2}}, 1\right) m(x)|u(x)|_{F_{x}}^{2}-(\lambda+C+1 / 2)\|u\|^{2} .
\end{aligned}
$$

Choosing, for example, $\lambda=-C-3 / 2$ in (35) we get the inequality (32) with $c_{1}=1$. Thus, $\left.\left(\widetilde{H}_{W, \Phi}-\lambda\right)\right|_{\Gamma_{c}(X, F)}$ with $\lambda=-C-3 / 2$ is a symmetric operator satisfying $\left(u,\left(\widetilde{H}_{W, \Phi}-\lambda\right) u\right) \geq\|u\|^{2}$, for all $u \in \Gamma_{c}(X, F)$. By Theorem X.26 in [26] we know that the essential self-adjointness of $\left.\left(\widetilde{H}_{W, \Phi}-\lambda\right)\right|_{\Gamma_{c}(X, F)}$ is equivalent to the following statement: if $v \in \Gamma_{\ell_{m}^{2}}(X, F)$ satisfies $\left(\widetilde{H}_{W, \Phi}-\right.$ $\lambda) v=0$, then $v=0$. Thus, by Lemma 6.2, the operator $\left.\left(\widetilde{H}_{W, \Phi}-\lambda\right)\right|_{\Gamma_{c}(X, F)}$ is essentially self-adjoint. Thus, $\left.\widetilde{H}_{W, \Phi}\right|_{\Gamma_{c}(X, F)}$ is essentially self-adjoint. 


\section{APPENDIX}

In this section we review some concepts from the theory of one-parameter semigroups of operators on Banach spaces. Our exposition follows Chapters I and II of [7]. A family of bounded linear operators $(T(t))_{t \geq 0}$ on a Banach space $\mathscr{X}$ is called a strongly continuous semigroup (or $C_{0}$-semigroup) if it satisfies the functional equation

$$
T(t+s)=T(t) T(s), \quad \text { for all } t, s \geq 0, \quad T(0)=I,
$$

and the maps $t \mapsto T(t) u$ are continuous from $\mathbb{R}_{+}$to $\mathscr{X}$ for all $u \in \mathscr{X}$. Here, $I$ stands for the identity operator on $\mathscr{X}$.

The generator $A: \operatorname{Dom}(A) \subset \mathscr{X} \rightarrow \mathscr{X}$ of a strongly continuous semigroup $(T(t))_{t \geq 0}$ on a Banach space $\mathscr{X}$ is the operator

$$
A u:=\lim _{h \rightarrow 0+} \frac{T(h) u-u}{h}
$$

defined for every $u$ in its domain

$$
\operatorname{Dom}(A):=\left\{u \in \mathscr{X}: \lim _{h \rightarrow 0+} h^{-1}(T(h) u-u) \text { exists }\right\} .
$$

By Theorem II.1.4 in [7], the generator of a strongly continuous semigroup is a closed and densely defined operator that determines the semigroup uniquely.

A linear operator $A$ on a Banach space $\mathscr{X}$ with norm $\|\cdot\|$ is called accretive if

$$
\|(\xi+A) u\| \geq \xi\|u\|,
$$

for all $\xi>0$ and all $u \in \operatorname{Dom}(A)$. In the literature on semigroups of operators, the term dissipative is used when referring to an operator $A$ such that $-A$ is accretive. If $A$ is a densely defined accretive operator, then $A$ is closable and its closure $\bar{A}$ is also accretive; see Proposition II.3.14 in [7].

We now give another description of accretivity. Let $\mathscr{X}^{*}$ be the dual space of $\mathscr{X}$. By the Hahn-Banach theorem, for every $u \in \mathscr{X}$ there exists $u^{*} \in \mathscr{X}^{*}$ such that $\left\langle u, u^{*}\right\rangle=\|u\|^{2}=\left\|u^{*}\right\|^{2}$, where $\left\langle u, u^{*}\right\rangle$ denotes the evaluation of the functional $u^{*}$ at $u$. For every $u \in \mathscr{X}$, we define

$$
\mathscr{J}(u):=\left\{u^{*} \in \mathscr{X}^{*}:\left\langle u, u^{*}\right\rangle=\|u\|^{2}=\left\|u^{*}\right\|^{2}\right\} .
$$

By Proposition II.3.23 of [7], an operator $A$ is accretive if and only if for every $u \in \operatorname{Dom}(A)$ there exists $j(u) \in \mathscr{J}(u)$ such that

$$
\operatorname{Re}\langle A u, j(u)\rangle \geq 0 .
$$

An operator $A$ on a Banach space $\mathscr{X}$ is called maximal accretive if it is accretive and $\xi+A$ is surjective for all $\xi>0$. There is a connection between maximal accretivity and self-adjointness of operators on Hilbert spaces: $A$ is a self-adjoint and non-negative operator if and only if $A$ is symmetric, closed, and maximal accretive; see Problem V.3.32 in [16].

A contraction semigroup $(T(t))_{t \geq 0}$ on a Banach space $\mathscr{X}$ is a semigroup such that $\|T(t)\| \leq 1$ for all $t \geq 0$, where $\|\cdot\|$ denotes the operator norm (of a bounded linear) operator $\mathscr{X} \rightarrow \mathscr{X}$. 
Generators of strongly continuous contraction semigroups are characterized as follows (Theorem II.3.5 in [7]):

Hille-Yosida Theorem. An operator A on a Banach space generates a strongly continuous contraction semigroup if and only if the following three conditions are satisfied:

(C1) $A$ is densely defined and closed;

(C2) $(0, \infty) \subset \rho(A)$, where $\rho(A)$ is the resolvent set of $A$;

(C3) $\left\|(\xi-A)^{-1}\right\| \leq \xi^{-1}$, for all $\xi>0$.

Finally, we note that if $A$ generates a strongly continuous contraction semigroup, then $-A$ is maximal accretive.

\section{ACKNOWLEDGMENT}

The authors are grateful to the anonymous referee for providing valuable suggestions and helping us improve the presentation of the material.

\section{REFERENCES}

1. Chung, F. R. K., Sternberg, S.: Laplacian and vibrational spectra for homogeneous graphs. J. Graph Theory. 16, 605-627 (1992)

2. Colin de Verdière, Y., Torki-Hamza, N., Truc, F.: Essential self-adjointness for combinatorial Schrödinger operators II-Metrically non complete graphs. Math. Phys. Anal. and Geom. 14, 21-38 (2011)

3. Colin de Verdière, Y., Torki-Hamza, N., Truc, F.: Essential self-adjointness for combinatorial Schrödinger operators II-Metrically non complete graphs. arXiv:1006.5778 v3

4. Colin de Verdière, Y., Torki-Hamza, N., Truc, F.: Essential self-adjointness for combinatorial Schrödinger operators III-Magnetic fields. Ann. Fac. Sci. Toulouse Math. (6) 20, 599-611 (2011)

5. Colin de Verdière, Y., Truc, F: Confining quantum particles with a purely magnetic field. Ann. Inst. Fourier (Grenoble) 60 (7), 2333-2356 (2010)

6. Dodziuk, J., Mathai, V.: Kato's inequality and asymptotic spectral properties for discrete magnetic Laplacians. In: Contemporary Mathematics, vol. 398, pp. 69-81. American Mathematical Society, Providence (2006)

7. Engel, K.-J, Nagel, R.: One-Parameter Semigroups for Linear Evolution Equations. Graduate Texts in Mathematics 194. Springer, Berlin (2000)

8. Folz, M.: Gaussian upper bounds for heat kernels of continuous time simple random walks. Electron. J. Probab. 16, 1693-1722 (2011)

9. Frank, R. L., Lenz, D., Wingert, D.: Intrinsic metrics for non-local symmetric Dirichlet forms and applications to spectral theory. J. Funct. Anal. 266, 4765-4808 (2014)

10. Güneysu, B., Keller, M., Schmidt, M.: A Feynman-Kac-Itô formula for magnetic Schrödinger operators on graphs. arXiv:1301.1304

11. Güneysu, B., Milatovic, O., Truc, F.: Generalized Schrödinger semigroups on infinite graphs. Potential Anal. 41, 517-541 (2014)

12. Haeseler, S., Keller, M.: Generalized solutions and spectrum for Dirichlet forms on graphs. In: Random Walks, Boundaries and Spectra. Progress in Probability, vol. 64, pp. 181-199. Birkhäuser, Basel (2011)

13. Hua, B., Keller, M.: Harmonic functions of general graph Laplacians. Calc. Var. Partial Differential Equations 51, 343-362 (2014)

14. Huang, X.: On stochastic completeness of weighted graphs. PhD thesis, Bielefeld (2011) 
15. Huang, X., Keller, M., Masamune, J., Wojciechowski, R. K.: A note on self-adjoint extensions of the Laplacian on weighted graphs. J. Funct. Anal. 265, 1556-1578 (2013)

16. Kato, T.: Perturbation Theory for Linear Operators. Springer-Verlag, Berlin (1980)

17. Kato, T.: $L^{p}$-theory of Schrödinger operators with a singular potential. In: Aspects of Positivity in Functional Analysis, R. Nagel, U. Schlotterbeck, M. P. H. Wolff (editors), pp. 63-78. North-Holland (1986)

18. Keller, M., Lenz, D.: Unbounded Laplacians on graphs: basic spectral properties and the heat equation. Math. Model. Nat. Phenom. 5 (4), 198-224 (2010)

19. Keller, M., Lenz, D.: Dirichlet forms and stochastic completneness of graphs and subgraphs. J. Reine Angew. Math. 666, 189-223 (2012)

20. Kenyon, R.: Spanning forests and the vector bundle Laplacian. Ann. Probab. 39, 1983-2017 (2011)

21. Masamune, J.: A Liouville property and its application to the Laplacian of an infinite graph. In: Contemporary Mathematics, vol. 484, pp. 103-115. American Mathematical Society, Providence (2009)

22. Masamune, J., Uemura, T.: Conservation property of symmetric jump processes. Ann. Inst. Henri Poincaré Probab. Stat. 47, 650-662 (2011)

23. Milatovic, O.: On $m$-accretivity of perturbed Bochner Laplacian in $L^{p}$ spaces on Riemannian manifolds. Integr. Equ. Oper. Theory 68, 243-254 (2010)

24. Milatovic, O.: Essential self-adjointness of magnetic Schrödinger operators on locally finite graphs. Integr. Equ. Oper. Theory 71, 13-27 (2011)

25. Milatovic, O., Truc, F.: Self-adjoint extensions of discrete magnetic Schrödinger operators. Ann. Henri Poincaré 15, 917-936 (2014)

26. Reed, M., Simon, B.: Methods of Modern Mathematical Physics II: Fourier Analysis, Self-Adjointness. Academic Press, New York (1975)

27. Singer, A., Wu, H.-T.: Vector diffusion maps and the connection Laplacian. Comm. Pure Appl. Math. 65, 1067-1144 (2012)

Department of Mathematics and Statistics, University of North Florida, Jacksonville, FL 32224 , USA

E-mail address: omilatov@unf.edu

Grenoble University, Institut Fourier, Unité mixte De Recherche CNRS-UJF 5582, BP 74, $38402-$ Saint Martin d'Hères Cedex, France

E-mail address: francoise.truc@ujf-grenoble.fr 